\title{
HIV and Aging in Mainland China: Implications for Control and Prevention Research
}

\author{
$\mathrm{Na} \mathrm{He}{ }^{1,2} \cdot$ Yingying Ding ${ }^{1} \cdot$ Jing $\mathrm{Li}^{1} \cdot$ Shiying Yuan ${ }^{1} \cdot$ Lulu Xu $^{1} \cdot$ Shijie Qiao ${ }^{1} \cdot$ Xiaohui Xu $^{1} \cdot$ Bowen Zhu $^{1} \cdot$ Ruizi Shi $^{1}$. \\ John P. Barile ${ }^{3} \cdot$ Frank Y. Wong ${ }^{1,3,4}$
}

Published online: 26 November 2019

(C) Springer Science+Business Media, LLC, part of Springer Nature 2019

\begin{abstract}
Purpose of Review The last 15 years have witnessed a dramatic change in HIV-related epidemiology amidst improvements in treatment and care in China. With proper treatment, HIV is now considered a chronic disease. As a consequence, many people living with HIV (PLWH) now present age-related comorbidities. We reviewed 13 topical issues concerning the epidemiology of aging with HIV in mainland China.

Results Many of aging-related issues associated with the biological and physical aspects of living with HIV addressed in mainland China are consistent with the global literature. Greater prevalence of age-related comorbidities among PLWH was observed. Beyond biological mechanisms associated with HIV infection and traditional risk factors, other factors play a vital role in the aging process among PLWH.

Summary A stronger focus on screening, prevention, and management of non-HIV co-morbidities among PLWH is now warranted. Macro-social factors need to be integrated into next generation of clinical and/or behavioral HIV research to inform disease progression and management as well as prevention.
\end{abstract}

Keywords Aging $\cdot \mathrm{HIV} \cdot$ Age-related diseases $\cdot$ Risk factors $\cdot$ China

\section{Introduction}

The biological definition of aging is the accumulation of changes in the cells and tissues associated with advancing age that increase the risks for diseases and eventual death [1,

$\mathrm{Na} \mathrm{He}$ and Yingying Ding are co-first authors.

This article is part of the Topical Collection on The Global Epidemic

$\mathrm{Na} \mathrm{He}$

nhe@fudan.edu.cn

$\triangle$ Frank Y. Wong

fwong@fsu.edu

1 Department of Epidemiology, School of Public Health, The Key Laboratory of Public Health Safety of Ministry of Education, Fudan University, Shanghai, China

2 Key Laboratory of Health Technology Assessment of Ministry of Health, Fudan University, Shanghai, China

3 Department of Psychology, University of Hawai'i at Mānoa, Honolulu, HI, USA

4 Center for Indigenous Nursing Research for Health Equity, Florida State University, Tallahassee, FL, USA
2]. An individual's biological age can differ markedly from their chronological age. The extent to which they differ varies widely by individual and largely attributable to developmental and genetic defects, the environment (e.g., living conditions), disease processes, and an inherent process known as "the aging process" [2]. This is particularly relevant to and of concern for people living with HIV (PLWH). The success of combination antiretroviral therapy (cART) has transformed HIV infection from a fatal to a chronic disease with life expectancy among PLWH getting closer to that of the general population. Meanwhile, these individuals present an increased prevalence of age-related co-morbidities [3]. However, the precise aging mechanisms among PLWH vs. those without HIV infection have yet to be fully understood [3].

The last 15 years have seen a dramatic change in HIV epidemiology amidst improvement in treatment and care in China. Notably, there is now a large number of PLWH who are ages 50 years or older. These individuals are likely to have increased odds of aging-related non-communicable chronic diseases (NCDs) and to have increased health care needs in the coming years [4]. In this article, we reviewed the literature on the epidemiology of aging with HIV in mainland China. For this review, we combed both the Chinese- and English- 
language scientific literature using the broad term "HIV and aging." This search has resulted in a total 13 topical issues being grouped under three broad themes prevalent in the HIV and aging literature, with the last two themes presented around the postulated model of the pathophysiology of aging and aging-related co-morbidities in HIV infection (Fig. 1). The themes include (1) aging profile of the HIV epidemic, (2) prevalence and incidence of aging-related chronic diseases, and (3) risk factors for aging-related chronic diseases.

For each topical issue, we first presented the latest empirical research, followed by a brief summary assessment. We then briefly addressed the challenges and opportunities of the next generation HIV control and prevention efforts in China.

\section{Aging Profile of the HIV Epidemic}

\section{Increasing Survival of PLWH}

Using data from the China national HIV epidemiology and treatment databases, Zhang et al. [5] examined the mortality rate of 145,484 PLWH aged 15 years or older who were eligible for cART between 1985 and 2009 . They found that the overall mortality decreased from 39.3 per 100 person-years (PY) in 2002 to 14.2 per $100 \mathrm{PY}$ in 2009 , with treatment coverage concomitantly increasing from almost zero to $63.4 \%$.
Dou et al. [6] conducted a retrospective cohort study with 368,449 cases on cART during 2002-2014. The 1-, 5-, and 10 -year survival rates were $92.2 \%, 80.5 \%$, and $69.6 \%$, respectively, among all PLWH, $97.9 \%, 89.8 \%$, and $81.0 \%$, respectively, for those with baseline CD4 count $>350$ cells $/ \mu \mathrm{L}$.

Another nationwide, retrospective, observational cohort study with 46,083 HIV-positive men and 22,563 HIV-positive women who initiated standard cART between January 1, 2010, and December 31, 2011, and were followed up to December 31, 2015 revealed that the all-cause mortality was lower in women than in men (2.34 vs. 4.03 deaths/100 PY; adjusted hazard ratio (HR), $0.72 ; 95 \%$ CI, $0.67-0.77 ; p<0.001$ ), and the 4year cumulative survival was $91.6 \%$ for women and $86.3 \%$ for men [7].

Collectively, these data demonstrate that the majority of PLWH on cART in China are able to rapidly achieve virological suppression with fairly good immunological recovery, which result in significant improvements in survival.

\section{Increasing Number and Proportion of Newly Reported Older Cases}

From 2010 to 2018, the annual number of newly reported PLWH aged 60 years or older increased by $>5$ folds from 5946 to 31,541 overall, from 4751 to 24,465 for older men, and from 1195 to 7076 for older women, respectively.
Fig. 1 A model of the pathophysiology of aging and aging-related co-morbidities associated with HIV infection

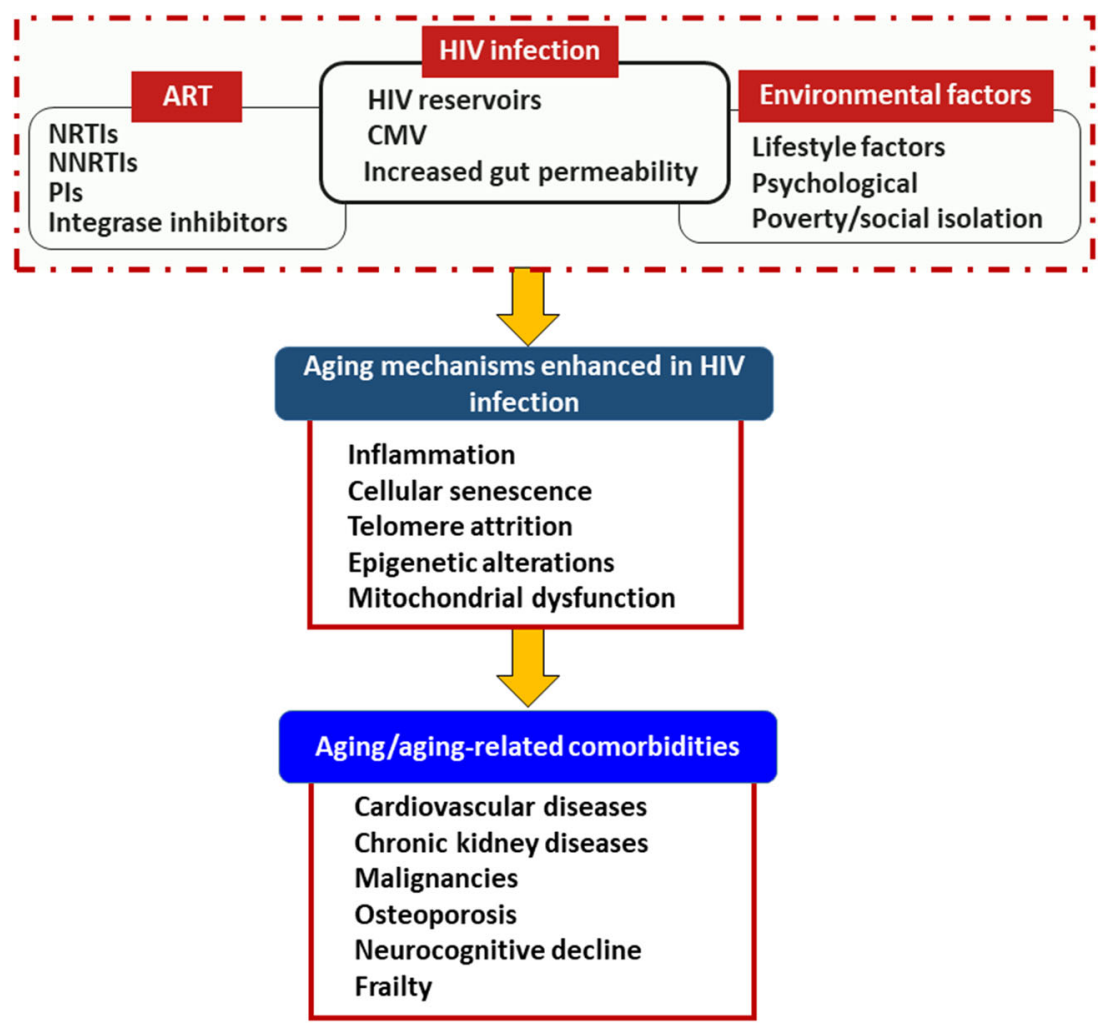


Correspondingly, the proportion of newly reported PLWH aged 60 years or older increased from $9.27 \%$ in 2010 to $21.22 \%$ in 2018, according to the statistics of Chinese National Center for AIDS and STD Control and Prevention. The majority of them were infected through heterosexual contacts, and very likely owing to low knowledge of HIV/AIDS and low awareness of HIV risks among the general older population in China [8]. In short, the HIV epidemic has increasingly affected older people, especially among older men in the past decade.

\section{Increasing Manifestation of Frailty and Fatigue}

Using a cross-sectional design, from June 2014 to May 2015, Ding et al. [9・•] consecutively enrolled 345 PLWH aged $\geq$ 40 years in Taizhou Prefecture of Zhejiang Province, who have registered with the Chinese National Information System for AIDS Prevention and Control (CNISAPC) and age, sex, and education frequency-matched HIV-negative controls in a 1:1 ratio by sex, education level, and 5-year age categories. Based on the Fried criteria [10], the authors found that PLWH were more likely to be frail $(6.1 \%$ vs. $0.6 \%)$ and prefrail $(28.1 \%$ vs. $11.9 \%)$ than HIV-negative individuals $(p<0.01)$. This association remained significant after adjustment for potential confounders (odds ratio [OR], 3.79). After additional adjustment for neurocognitive impairment and depressive and insomnia symptoms, this association remained significant but attenuated (OR, 2.16). Furthermore, in the PLWH, neurocognitive impairment, depressive, and insomnia symptoms were independently associated with prefrailty/frailty. These findings suggest that neurocognitive impairment and depressive and/or insomnia symptoms may account for a higher prevalence of prefrailty/frailty in Chinese PLWH. To our knowledge, this is the only published study specifically designed to investigate prevalence and correlates of prefrailty/ frailty among PLWH in China.

PLWH report a high prevalence rate of fatigue but none of studies have directly compared them with HIV-negative individuals. Using data from the CNISAPC, Wang et al. [11] retrospectively examined the occurrence and risk factors of self-reported fatigue among a cohort of HIV patients in Eastern China in the first 6 months after receiving cART. Overall, 22.4\% (261/1163) reported fatigue within the first 6 months after receiving cART, which was negatively associated with higher education, higher baseline CD4 cell count, and overweight but positively associated with $\geq 50$ years old at initiation of ART (vs. $<30$ years old), underweight at baseline, and use of efavirenz in the first-line regimen. These findings suggest that initiation of cART at a high CD4 count and a healthy body mass index are assumed to experience less fatigue associated with cART than older adults and those who are underweight.
Using a mixed-method study design with qualitative indepth interviews with 19 HIV-positive women and a quantitative cross-sectional audio computer-assisted self-interviews with a convenience sample of 107 PLWH ( $84 \%$ were male) in Shanghai, Chen et al. [12] noted that self-reported stress associated with living the disease, sleep disturbance, and fatigue were the major themes emerged from the in-depth interviews, and moderate fatigue severity $(M=5.24, \mathrm{SD}=2.27)$ measured with Fatigue Scale [13] in the cross-sectional data. These findings suggest that the relationship between stress and fatigue might be largely (53\%) mediated through sleep disturbance.

\section{Prevalence and Incidence of Aging-Related Chronic Diseases}

\section{Cardiovascular Diseases}

Guo et al. [14] retrospectively analyzed risks associated with cardiovascular diseases (CVDs) among 973 treatment-naïve PLWH ( $72 \%$ men) with a mean age of $36.0 \pm 10.2$ years enrolled in two multi-center clinical trials. The prevalence of hypertension, diabetes, and obesity was $8.4 \%, 4.6 \%$ and $1.0 \%$, respectively. Over $65 \%$ of the patients had at least one CVD risk factor. The prevalence of 10 -year CVD risk $\geq 10 \%$ was $4.5 \%$ based upon the Framingham Risk Score (FRS) and was 3.3\% based upon Data Collection on Adverse Events of Anti-HIV Drugs risk score [14].

In another multi-center cross-sectional study of 538 ARTnaïve PLWH ( 399 men) with a mean age of $36.5 \pm 10.0$ years, the prevalence of hypertension and glycometabolism abnormities were $3.2 \%$ and $3.0 \%$, respectively [15].

Ding et al. [16] examined the prevalence of hypertension among 345 PLWH and 345 HIV-negative individuals aged $\geq$ 40 years who were frequency matched on age, gender, and education with a mean age of 52.7 years, where $77.7 \%$ were men in both groups and $87 \%$ of PLWH were on cART. Results showed that hypertension prevalence was lower in PLWH than in HIV-negative participants ( $23.8 \%$ vs. $31.9 \%$; $p=0.011$ ), but this association was not significant after adjusting for potential confounders. Among PLWH, older age, overweight, with a family history of CVDs, and nadir CD4 count $<50$ cells $/ \mu \mathrm{L}$ were independently associated with hypertension.

Lin et al. [17••] preformed a cross-sectional evaluation of baseline data from the Comparative HIV and Aging Research in Taizhou (CHART) Cohort. They found that 480 of 1425 (36.1\%; 95\% CI, 33.6-38.6) PLWH and 784 of 2850 (27.5\%; 95\% CI, 25.9-29.2) HIV-negative individuals had subclinical atherosclerosis as assessed by carotid artery intima-media thickness (cIMT) $(p<0.0001)$, and these patterns remained significant (adjusted OR, 1.72; 95\% CI, 1.47-2.01) in the adjusted model. Significant negative interaction between HIV-positive infection serostatus and age on subclinical 
atherosclerosis was observed $(p<0.0001)$. Age-, sex-, and FRS-adjusted OR and 95\% CI for the age groups of 18-29, $30-44,45-59$, and $60-75$ years were 8.84 (4.50-17.34), 2.09 (1.59-2.74), 1.54 (1.19-1.98), and 1.04 (0.75-1.44), respectively. Among PLWH, none of HIV-specific variables was statistically significantly associated with cIMT estimates except for being ART-naïve.

Collectively, these findings are consistent with the global literature that PLWH have higher rates of CVDs.

\section{Diabetes Mellitus}

In a cross-sectional assessment of newly diagnosed and ARTnaïve PLWH (75.67\% men with a median age of 40) in 2006 from 10 provinces and municipalities, $19.99 \%$ had hyperglycemia, $9.47 \%$ had impaired fasting glucose (IFG), and $10.52 \%$ had diabetes [18]. Older age, lower CD4 count, and minority ethnicity are associated with increased risk of diabetes in this sample. Ye et al. [19] recently reported that the prevalence of diabetes was $11.4 \%$ among a total of 4376 PLWH on cART with the average age of $(43.7 \pm 10.1)$ years. Aged 40 years or above, being male, HCV positive, baseline body mass index $\geq 24.0 \mathrm{~kg} / \mathrm{m}^{2}$, and elevated triglyceride $\geq$ $1.70 \mathrm{mmol} / \mathrm{L}$ were associated with the presence of diabetes.

In another study, Cheng et al. [20] reported that the prevalence of hyperglycemia was $15.7 \%$ among 504 AIDS patients who had received ART for at least 3 months and was significantly associated with older age, family history of diabetes, overweight, nadir CD4 cell counts of 50-199 cells $/ \mu \mathrm{L}$, and less than 50 cells $/ \mu \mathrm{L}$. Unfortunately, little is known about the incidence of diabetes mellitus or hyperglycemia. It is noted that these studies report a much higher prevalence of diabetes than those among ART-naïve PLWH in studies by Gau et al (4.6\%) [14] and Cao et al. (3.0\%) [15].

A pooled longitudinal data from a multi-center clinical trial of cART regimens between 2009 and 2010 across mainland China indicates that the incidence densities of diabetes defined as fasting glucose level $\geq 7.0 \mathrm{mmol} / \mathrm{L}$ and IFG were 2.62 and 35.64 per 100 PY, respectively, and were significantly associated with advanced age (adjusted HR, 1.03; 95\% CI, 1.011.04], hepatitis B virus co-infection (adjusted HR, 1.59; 95\% CI, 1.06-2.38), and baseline fasting glucose (adjusted HR, $1.28,95 \%$ CI, 1.00-1.63) [21].

In brief, the prevalence of diabetes mellitus among PLWH varies significantly by the study sample, geographical regions, and ART status.

\section{Kidney Disease}

A hospital-based multi-center cross-sectional study reported that the prevalence of chronic kidney disease (CKD) was $16.1 \%$ among 538 ART-naïve PLWH, who tended to be older with HCV co-infection and plasma HIV-1 viral load 100,000
copies/mL [15]. Another study clinically described the epidemiology, outcomes, and risk factors of acute kidney injury (AKI) among 225 PLWH who were admitted to the intensive care unit at Beijing Ditan Hospital from June 2005 to May 2017 [22]. The incidences of no-AKI, AKI stage 1, AKI stage 2, and AKI stage 3, were 46.2\% (104), $19.1 \%$ (43), 8.4\% (19), and 26.2\% (59), respectively, and severe AKI (stages 2-3) was an important predicator for the 60-day mortality with an OR of 4.23 [22].

In another study, Ding et al. [23] retrospectively examined renal function and progression to CKD among 5533 PLWH on cART between 2004 and 2016 in Yunnan Province of Southwestern China. The investigators found that progression to CKD occurred in $2.3 \%$ patients' overall and was more frequently observed in patients with baseline estimated glomerular filtration rate (eGFR) $<60$ and $60-<90 \mathrm{~mL} / \mathrm{min} /$ $1.73 \mathrm{~m}^{2}$ (4.6\% and $5.1 \%$, respectively), and older age was consistently independently associated with CKD [23].

Globally, HIV-associated nephropathy (HIVAN) was a leading cause of HIV-associated kidney diseases during the early HIV epidemic, but it has largely declined in the era of viral suppressive cART [24]. However, PLWH on treatment continue to have much higher rates of CKD than HIVnegative individuals $[25,26]$, increasing their risks for CVDs, end-stage renal disease, and premature mortality [27].

\section{Liver Fibrosis}

HIV alone can induce liver fibrosis, whereas co-infections with other types of hepatitis accelerate the progression of related liver diseases, including liver fibrosis, cirrhosis, and death due to end-stage liver diseases including liver cancer. Fortunately, cART is associated with an overall reduction in liver fibrosis regardless of HBV and HCV co-infection status, which mostly occurs during the first 3 months of treatment and maintains for long periods. CD4 recovery and HIV RNA suppression are protective factors for liver fibrosis progression; nonetheless, older age, male, $\mathrm{HCV}$ co-infection, and TDF use are risk factors $[28,29]$. These findings suggest that early treatment can mitigate the development or progression of liver fibrosis.

\section{Neurocognitive Impairment and Disorders}

Heaton et al. [30] administered a comprehensive neuropsychological (NP) test battery to 203 HIV-positive and 198 HIV-negative former plasma donors in a rural area of Anhui province, Central China. Using a global summary score, NP impairment was found in $34.2 \%$ of the HIV mono-infected group and $39.7 \%$ of the co-infected group, as compared with $12.7 \%$ of the HIV-negative controls $(p<0.001)$.

In a second study, these investigators further examined the incidence of cognitive decline over 1 year among 192 PLWH and 101 demographically comparable HIV-negative former 
plasma donors in the same area, of whom $56 \%$ of HIVpositive group was on cART at entry and $60.9 \%$ at followup. The 1-year cumulative incidence of cognitive decline was significantly higher among PLWH than HIV-negative individuals $(27 \%$ vs. $5 \%)$, was predictable by AIDS status, lower nadir CD4, and worse processing speed at baseline, and was associated with lower current CD4 cell count and failure of viral suppression on cART at follow-up [31].

Zhao et al. [32] assessed cognitive disorders in $230 \mathrm{HIV} /$ AIDS patients and 99 healthy individuals in Guangxi province, Southwestern China. With 13 tests from 12 reliable and valid neuropsychological instruments (the digit symbol test, trail making test, arithmetic scores, digit span, wood puzzle, immediate visual memory, visual memory, Stroop test, vocabulary fluency, conceptual fluency, and the Wisconsin Card Sorting Test), 86 patients (37.39\%) had HIV-associated neurocognitive disorders (HAND). Among them, 42 (18.27\%) had HIV-related neurocognitive impairment (ANI), 25 (18.87\%) had HIVrelated mild neurocognitive disorder (MND), and 19 (8.26\%) had HIV-associated dementia (HAD).

In another study, Zhang et al. [33] reported that $37 \%$ of 134 PLWH from three different regions had HAND measured by the International HIV Dementia Scale (IHDS) and NP test; suggesting that over one-third of PLWH will develop a HAND.

In a study of neurocognitive impairment (NCI) screening using the IHDS and Chinese version of Mini-Mental State Examination (MMSE) among 345 PLWH and 345 HIVnegative participants aged at least 40 years in Zhejiang Province, Eastern China, Ding et al. [34] reported that PLWH had higher prevalence of NCI than HIV-negative individuals $(46.7 \%$ vs. $15.1 \%$ for IHDS using cut-off of $\leq 10$; $17.1 \%$ vs. $2.6 \%$ for MMSE). Interestingly, the investigators observed significant main effects of HIV and age on IHDS and MMSE composite scores and significant interaction effects between HIV and age on motor speed, orientation, registration, and recall, which were mainly attributed to the inferior performance of PLWH aged over 60 years.

Most recently, a study of NCI screening with MMSE among 669 PLWH (223 women) and 1338 HIV-negative controls (446 women) frequency matched for age, sex, and education revealed that while $12.4 \%$ of PLWH were detected to have NCI compared with $3.4 \%$ of the HIV-negative controls $(p<0.001)$, the NCI prevalence was higher among women than men in the HIV-positive group $(16.1 \%$ vs. $10.5 \%)$ but not the HIV-negative group (4.3\% vs. $3.5 \%$ ) [35].

Collectively, these findings are consistent with the global literature that PLWH has a higher rate of NCI or neurocognitive disorders.

\section{Malignant Tumors}

An early clinical epidemiological study of 3554 PLWH (45\% were female) with 11,072 person-years follow-up from 2004 to
2008 in Hubei Province in Central China identified 63 cancer cases with approximately 50\% (32) diagnosed with AIDSdefining cancers (ADCs). Non-Hodgkin's lymphoma, cervical cancer, and liver cancer are most common cancers in this sample [36]. Yang et al. [37] investigated cancer morbidity and mortality among 1946 PLWH in a hospital specialized in infectious diseases in Beijing from 2008 to 2013. Among them, $7.7 \%$ (149 cases) had concurrent cancer at their first hospital admission including 33.6\% (50 cases) having ADCs and 66.4\% (99 cases) having non-AIDS-defining cancers (NADCs). The most prevalent NADCs were Hodgkin's lymphoma, gastrointestinal cancer, liver cancer, and lung cancer. Patients who did not accept ART were more likely to suffer from cancer, and if diagnosed with cancer, had lower survival rate.

A recent meta-analytic review [38] based on 102 articles reveals that the prevalence of major cancer risk factors is high among PLWH [38]. Overall, the summary prevalence estimate (sPrev) and 95\% CI for each risk factor among PLWH were the following: $41.1 \%$ (95\% CI, 35.3-46.9\%) for current smoking, 30.3\% (95\%CI, 23.3-37.4\%) for current alcohol consumption, $24.4 \%$ (95\% CI, 14.7-30.2\%) for overweight and obesity, $12.5 \%$ (95\% CI, 10.6-14.3\%) for hepatitis B virus infection, $29.1 \%$ (95\% CI, 23.6-34.5\%) for hepatitis C virus infection, $33.9 \%$ (95\% CI, $24.3-43.5 \%$ ) for high-risk human papillomavirus infection from cervical samples, and 78.6\% (95\% CI, 69.4-87.7\%) from anal samples; $2.7 \%$ (95\% CI, 0.7-4.7\%) for Epstein-Barr virus (EBV) immunoglobulin M (IgM) positivity, $94.7 \%$ (95\% CI, 90.7-98.8\%) for EBV IgG positivity, and $25.6 \%$ (95\% CI, 12.4-38.8\%) for EBV DNA positivity; $14.9 \%$ (95\% CI, 12.4-17.4\%) for human herpes virus 8 infection.

Cancer is now one of the leading causes of death among PLWH with higher incidence of both ADCs and NADCs compared with the general population in many countries [39, 40]. However, research on PLWH with concurrent cancer is rare in China.

\section{Risk Factors for Aging-Related Chronic Diseases}

\section{Lifestyle Risk Factors}

Smoking, alcohol use, and obesity are common risk factors for aging-related chronic diseases such as CVDs and cancer. As systematically reviewed by Jin et al. [38], the sPrev among Chinese PLWH was $41.1 \%$ for current smoking and $30.3 \%$ for current alcohol drinking.

PLWH tend to lose weight after viral infection and therefore have lower weight than normal individuals. However, this might be changing after cART. According to Jin et al., the sPrev for overweight or obesity was $22.4 \%$ among Chinese PLWH, considerably lower than overweight or obesity rates 
in the general Chinese population (42.6\%) [38]. Ding et al. [16] reported that PLWH were often less overweight and obese (20.9\% and $4.3 \%$ ) compared with HIV-negative individuals $(40.6 \%$ and $5.8 \%)(p<0.001)$. In a recently completed large cross-sectional study, $23.4 \%$ (334/1425) of PLWH had $\mathrm{BMI} \geq 24 \mathrm{~kg} / \mathrm{m}^{2}$, compared with $47.9 \%(665 / 1366 / 2850)$ among HIV-negative participants, whereas $48.9 \%$ of HIVpositive group vs. $47.8 \%$ of HIV-negative group had abdominal obesity which was measured by waist-to-hip ratio (WHR) (the cutoff of WHR for abdominal obesity is 0.9 for men and 0.85 for women) [17]. Despite obesity is currently not a major current health issue among PWLH in China, it is expected to increase as since 2016, China updated its ART guideline and recommended initiation of ART for all PLWH, regardless of CD4 count.

Collectively, these findings are consistent with the global literature. However, the scientific breadth and depth regarding the behavioral and/or physical risks and HIV and aging among PLWH in China are scarce.

\section{Sleep Disturbance}

Aging and PLWH often suffer from sleep disturbance and insomnia which are usually associated with frailty, shorter telomere length, and aging-related NCDs such as NCIs [9, 41 •]. Chen et al. [12] initially examined sleep disturbance using General Sleep Disturbance Scale (GSDS) among a convenient sample of 107 PLWH (84\% were male). The investigators found that $40 \%$ of the participants reported clinically significant sleep disturbances (GSDS $>3$ ) with an average of 3 nights of disturbed sleep in the past week $(M=2.87, \mathrm{SD}=1.21)$.

In another study, Ren et al. [42] conducted a cross-sectional survey to identify prevalence and correlates of sleep quality complications using the Spiegel scale with 237 PLWH recruited from March to August in 2013 at HIV-designated hospitals in Heilongjiang Province, Northeastern China. Among them, $32.1 \%$ were negatively affected by sleep disturbances in which nighttime dreams and waking up were the most serious complications. In a third study, Huang et al. [43] evaluated the prevalence and factors associated with sleep disturbance using the Pittsburgh Sleep Quality Index (PSQI) with 4103 PLWH on ART at 20 AIDS clinics. Results revealed $43.1 \%$ of PLWH and were significantly associated with psychological factors including anxiety and depression, sociodemographic factors including being MSM, single, higher education, and clinical factors including suboptimal adherence, regimen switching, and antidepressant use. Last but not least, insomnia symptoms assessed by Jenkins Sleep Problems Scale, poor sleep quality assessed by PSQI, and long sleep duration ( $\geq 9 \mathrm{~h}$ ) were observed among $23.7 \%, 24.1 \%$, and $16.1 \%$ of HIV-positive vs. $19.8 \%, 19.9 \%$, and $8.7 \%$ of HIV-negative individuals in the baseline assessment of CHART Cohort, China [44].
In brief, the heterogeneity in the prevalence of sleep disturbance or insomnia reflects the variation in measurement scales and geographical regions.

\section{Dietary and Nutrition}

Shan et al. [45] examined food intake frequency among 278 PLWH who have been on ART for at least 1 year in a rural prefecture of Yunnan Province, Southwestern China. These investigators reported that only the average intake of cereals and beans/nuts per day met the recommended quantity by the Dietary Guidelines for Chinese Residents, and the average intake of vegetables, aquatic products, eggs, milk, and fruits per day was insufficient. Scenarios are more dire for older patients who got HIV infection through plasma donation or injection drug use and mostly co-infected with $\mathrm{HCV}$ at early epidemic stage in the late 1980s through 1990s, since coinfection with HIV-1 could alleviate iron accumulation in patients with chronic HCV infection [46].

Ji et al. [47] implemented a community-based "planting and eating soybean" project to increase soybean protein in the diet of PLWH in a resource-limited rural area in Anhui Province in Central China. Participants were encouraged to eat soy food every day after harvest. During the 3-month intervention period, $94 \%$ of the participants had eaten soy food at least 3 times a week and $96 \%$ of them ate $100 \mathrm{~g}$ each time. After eating soy food, $93 \%$ felt better, $86 \%$ reported less sickness, $61.3 \%$ had higher total blood protein and blood white protein, $58.1 \%$ had higher blood hemoglobin, and $54.8 \%$ had higher $\mathrm{CD} 4$ count.

Unfortunately, nutritional knowledge, attitudes, and behaviors among Chinese PLWH are generally deficient and need improvement. For example, in a cross-sectional survey on 172 homosexual PLWH in Chongqing, a metropolitan city in Southwestern China, the average score on the knowledge quiz was $51.8 \%$, eating habits did not meet the nutritional requirements, and $59.3 \%$ did not eat breakfast every day [48].

Many PLWH in China are living in rural areas, are relatively poor, and lack sufficient protein or other nutrients in their diets. These studies speak to the urgent need to integrate nutrition in HIV treatment and care in China.

\section{Other Biological and Genetic Risk Factors}

HIV infection is associated with ongoing activation of the immune system and persistent inflammation, despite virological suppression by cART [49]. These are key driving forces in the loss of CD4+ T cells, progression to AIDS, and development of non-HIV-related but aging-related chronic complications such as CVD and certain cancers [49-51]. An early loss of gut mucosal integrity, the following microbial translocation, the pro-inflammatory cytokine milieu, and co- 
infections are all factors contributing to the ongoing immune activation and sustained inflammation [52].

Genetic factors play important roles in occurrence and development of various NCDs. Very recently, Zhao et al. [53] demonstrate the association between mitochondrial DNA (mtDNA) haplogroups and metabolic syndrome (MetS) in PLWH in China, and in particular, the Asian-specific mtDNA haplogroups G and N9 may confer higher risk for the development of MetS in PLWH.

However, no studies have been reported to explore the relationship between inflammation or inflammatory biomarkers and NCDs in Chinese HIV/AIDS patients. The role of genetic factors on HIV risks in among people of Asian descent warrant additional research.

\section{Conclusions}

In this review, we present a relatively comprehensive, though not exhaustive, coverage of HIV and aging among PLWH in mainland China. Overall, many of the aging-related issues associated with the biological and physical aspects of living with HIV addressed here are consistent with the global literature. Efforts on screening, prevention, and management of non-HIV co-morbidities are now warranted. Moreover, due to the scant literature, we did not cover macro-social factors on HIV and aging. Nonetheless, some of the research reviewed here do provide an opportunity to speculate what (and how) macro-social factors might play a role in the daily lives of PLWH in China. We briefly address two issues pertaining to these macro-social factors.

China does not have the resources to develop a comprehensive and robust system to promote the mental health among its populace. In the case of PLWH, their mental health is often not addressed. Many of these individuals attribute their sleepless and fatigue to stress. No doubt part of the physical conditions is due to living with the disease; nonetheless, the global literature unequivocally documents that depression (a mental health issue) is a common condition among many PLWH [54]. At the present time, there is limited program addressing depression among PLWH in China [55]. Perhaps, a systematic self-management intervention to decrease perceived stress could be developed and implemented in China's resourcelimited mental health settings to reduce sleep disturbance and fatigue - if not directly addressing depression.

As part of this dialog on the continuum addressing mental health treatment and care in China, health education and promotion should occupy an important role in working with PLWH. For example, nutrition education needs to be an integral part of the national HIV treatment and care strategies. Other efforts (e.g., smoking cessation and reduction in alcohol use) that could promote healthy lifestyles targeting PLWH also need to be part of this plan. These coordinated strategies are crucial to slow the stampede of the HIV-aging process.

Last but not least, with HIV as a chronic disease, it is necessary to re-focus some of the research agendas and/or priorities in China. Aging is more than just a biological process; it is also a social process. For example, there is an unequivocal body of global research documenting that negative macrosocial factors, such poverty [56] and social isolation [57, 58], are contributing to a range of health disparities including living with HIV $[59,60]$. China is lacking behind other countries in the study of the effect of macro-social determinants on the HIV-and-aging continuum. For instance, we know little about how and in what way living conditions (e.g., rural vs. urban) and stress induced by discrimination (e.g., due to HIV, sexual orientation, or both) affect the aging process among PLWH in China. In brief, it would be a wise investment to integrate some of the macro-social factors into clinical and/or behavioral HIV research.

Funding Information This research was supported by grants from the National Natural Science Foundation of China (81773485, 81872671, 81803291 ) and the China National Science and Technology Major Projects on Infectious Diseases (2018ZX10721102-004) and partially supported by Shanghai Municipal Health and Family Planning Commission (GWTD2015S05).

\section{Compliance with Ethical Standards}

Conflict of Interest The authors declare that they have no conflicts of interest.

Human and Animal Rights and Informed Consent This article does not contain any studies with human or animal subjects performed by any of the authors.

\section{References}

Papers of particular interest, published recently, have been highlighted as:

- Of importance

- Of major importance

1. Harman D. Aging: prospects for further increases in the functional life span. Age. 1994;17:119-46.

2. Harman D. Aging: overview. Ann N Y Acad Sci. 2001;928:1-21.

3. Pathai S, Bajillan H, Landay AL, High KP. Is HIV a model of accelerated or accentuated aging? J Gerontol A Biol Sci Med Sci. 2014;69(7):833-42.

4. Althoff KN, Smit M, Reiss P, Justice AC. HIV and ageing: improving quantity and quality of life. Curr Opin HIV AIDS. 2016;11(5): 527-36.

5. Zhang F, Dou Z, Ma Y, Zhang Y, Zhao Y, Zhao D, et al. Effect of earlier initiation of antiretroviral treatment and increased treatment coverage on HIV-related mortality in China: a national observational cohort study. Lancet Infect Dis. 2011;11(7):516-24. 
6. Dou Z, Zhang F, Zhao Y, Jin C, Zhao D, Gan X, et al. Progress on China's national free antiretroviral therapy strategy in 2002-2014 [article in Chinese]. Zhonghua Liu Xing Bing Xue Za Zhi. 2015;36(12):1345-50.

7. Chen M, Dou Z, Wang L, Wu Y, Zhao D, Gan X, et al. Gender differences in outcomes of antiretroviral treatment among HIVinfected patients in China: a retrospective cohort study, 2010 2015. J Acquir Immune Defic Syndr. 2017;76(3):281-8.

8. Wang C, Guo Q, Han Z, Chen D. Awareness rate of HIV/AIDS core knowledge among the elderly: a meta-analysis. Chin J AIDS \& STD. 2019;2:148-52.

9.• Ding Y, Lin H, Liu X, Wong FY, Sun YV, Marconi VC, et al. Higher prevalence of frailty among a sample of Chinese HIVinfected middle-aged and older adults is associated with neurocognitive impairment and depressive symptoms. J Infect Dis. 2017;215(5):687-69 This is the first study to report a higher prevalence of prefrailty and frailty among PLWH compared with similar but uninfected individuals in China and further indicate such association is partly explained by neurocognitive impairment and depressive symptoms.

10. Fried LP, Tangen CM, Walston J, Newman AB, Hirsch C, Gottdiener J, et al. Cardiovascular health study collaborative research group. Frailty in older adults: evidence for a phenotype. J Gerontol A Biol Sci Med Sci. 2001;56(3):146-56.

11. Wang K, Lin H, Li L, Wu Q, Shen W, Liu X, et al. Low body mass index and efavirenz use are independently associated with selfreported fatigue in HIV-infected patients. AIDS Care. 2019;31(4): 513-8.

12. Chen WT, Shiu CS, Yang JP, Lee SY, Lee TS, Simoni JM, et al. Fatigue and sleep disturbance related to perceived stress in Chinese HIV-positive individuals: a mixed methods study. J AIDS Clin Res. 2013;4(6): 15524.

13. Lee KA, Hicks G, Nino-Murcia G. Validity and reliability of a scale to assess fatigue. Psychiatry Res. 1991;36(3):291-8.

14. Guo F, Hsieh E, Lv W, Han Y, Xie J, Li Y, et al. Cardiovascular disease risk among Chinese antiretroviral-naïve adults with advanced HIV disease. BMC Infect Dis. 2017;17(1):287.

15. Cao Y, Gong M, Han Y, Xie J, Li X, Zhang L, et al. Prevalence and risk factors for chronic kidney disease among HIV-infected antiretroviral therapy-naïve patients in mainland China: a multicenter cross-sectional study. Nephrology (Carlton). 2013;18(4):307-12.

16. Ding Y, Lin H, Liu X, Zhang Y, Wong FY, Sun YV, et al. Hypertension in HIV-infected adults compared with similar but uninfected adults in China: body mass index-dependent effects of nadir CD4 count. AIDS Res Hum Retrovir. 2017;33(11):1117-25.

17.• Lin H, Ding Y, Ning C, Qiao X, Chen X, Chen X, et al. Agespecific associations between HIV Infection and carotid artery intima media thickness in China: a cross-sectional evaluation of baseline data from the CHART Cohort. Lancet HIV. Published Online October 18, 2019. https://doi.org/10.1016/S2352-3018(19)302 S2352-3018(19)30263-2. This is the first large-scale cross-sectional survey to demonstrate a higher prevalence of subclinical atherosclerosis among PLWH compared with age and sex frequency-matched HIV-negative individuals in China, and such differences are more obvious at younger ages.

18. Shen Y, Wang Z, Liu L, Zhang R, Zheng Y, Lu H. Prevalence of hyperglycemia among adults with newly diagnosed HIV/AIDS in China. BMC Infect Dis. 2013;13:79.

19. Ye RH, Li J, Yao ST, Wang JB, Cao DD, Zhang YD, et al. Prevalence and related factors on diabetes among HIV/AIDS receiving antiretroviral therapy in Dehong Dai and Jingpo Autonomous Prefecture. Zhonghua Liu Xing Bing Xue Za Zhi. 2019;40:654 $\mu$.

20. Cheng W, Wu Y, Dou Z, Ma Y, Sun L, Li Z, et al. The prevalence and relevant factors of hyperglycemia in AIDS patients receiving antiretroviral therapy in a single center. Zhonghua Nei Ke Za Zhi. 2014;53(6):446-9.

21. Zhang C, Chow FC, Han Y, Xie J, Qiu Z, Guo F, et al. Multicenter cohort study of diabetes mellitus and impaired fasting glucose in HIV-infected patients in China. J Acquir Immune Defic Syndr. 2015;68(3):298-303.

22. Kooij KW, Vogt L, Wit FWNM, van der Valk M, van Zoest RA, Goorhuis A, et al. Higher prevalence and faster progression of chronic kidney disease in human immunodeficiency virusinfected middle-aged individuals compared with human immunodeficiency virus-uninfected controls. J Infect Dis. 2017;216(6): 622-31.

23. Ding Y, Duan S, Ye R, Yao S, Cao D, Yang Y, et al. Effects of aging, baseline renal function and stage of HIV infection on post-treatment changes in renal function among HIV -infected patients: a retrospective cohort study. HIV Med. 2019;20(8):591-600.

24. Cohen SD, Kopp JB, Kimmel PL. Kidney diseases associated with human immunodeficiency virus infection. N Engl J Med. 2017;377(24):2363-74.

25. Schouten J, Wit FW, Stolte IG, Kootstra NA, van der Valk M, Geerlings SE, et al. Cross-sectional comparison of the prevalence of age-associated co-morbidities and their risk factors between HIV-infected and uninfected individuals: the AGEhIV cohort study. Clin Infect Dis. 2014;59(12):1787-97.

26. Coresh J, Turin TC, Matsushita K, Sang Y, Ballew SH, Appel LJ, et al. Decline in estimated glomerular filtration rate and subsequent risk of end-stage renal disease and mortality. JAMA. 2014;311(24): 2518-31.

27. Pu L, Liu J, Luo Y, Zeng H, Guo H, Hao J, et al. Acute kidney injury in Chinese HIV-infected patients: a retrospective analysis from the intensive care unit. AIDS Patient Care STDs. 2018;32(10):381-9.

28. Li Y, Xie J, Han Y, Wang H, Lv W, Guo F, et al. Combination antiretroviral therapy is associated with reduction in liver fibrosis scores in HIV-1-infected subjects. Medicine (Baltimore). 2016;95(2):e2660.

29. Ding Y, Duan S, Ye R, Yang Y, Yao S, Wang J, et al. More improvement than progression of liver fibrosis following antiretroviral therapy in a longitudinal cohort of HIV-infected patients with or without HBV and HCV co-infections. J Viral Hepat. 2017;24(5): 412-20.

30. Heaton RK, Cysique LA, Jin H, Shi C, Yu X, Letendre S, et al. Neurobehavioral effects of HIV infection among former plasma donors in rural China. J Neuro-Oncol. 2008;14(6):536-49.

31. Cysique LA, Letendre SL, Ake C, Jin H, Franklin DR, HIV Neurobehavioral Research Center group, et al. Incidence and nature of cognitive decline over 1 year among HIV-infected former plasma donors in China. AIDS. 2010;24(7):983-90.

32. Zhao T, Wei B, Long J, Tang X, Zhou M, Dang C. Cognitive disorders in HIV-infected and AIDS patients in Guangxi. China J Neurovirol. 2015;21(1):32-42.

33. Zhang Y, Qiao L, Ding W, Wei F, Zhao Q, Wang X, et al. An initial screening for HIV-associated neurocognitive disorders of HIV-1 infected patients in China. J Neuro-Oncol. 2012;18(2):120-6.

34. Ding $\mathrm{Y}$, Lin $\mathrm{H}$, Shen $\mathrm{W}, \mathrm{Wu} \mathrm{Q}$, Gao M, He N. Interaction effects between HIV and aging on selective neurocognitive impairment. J NeuroImmune Pharmacol. 2017;12(4):661-9.

35. Qiao X, Lin H, Chen X, Ning C, Wang K, Shen W, et al. Sex differences in neurocognitive screening among adults living with HIV in China. J Neurovirol. 2019;25(3):363-71 This study in China demonstrates that PLWH perform worse on cognitive measures compared with HIV-negative individuals, and sex difference is observed in HIV+ group only.

36. Zhang YX, Gui XE, Zhong YH, Rong YP, Yan YJ. Cancer in cohort of HIV-infected population: prevalence and clinical characteristics. J Cancer Res Clin Oncol. 2011;137:609-14. 
37. Yang J, Su S, Zhao H, Wang D, Wang J, Zhang F, et al. Prevalence and mortality of cancer among HIV-infected patients in Beijing. China BMC Infect Dis. 2016;16:82.

38. Jin ZY, Liu X, Ding YY, Zhang ZF, He N. Cancer risk factors among people living with HIV/AIDS in China: a systematic review and meta-analysis. Sci Rep. 2017;7:4890.

39. Shiels MS, Engels EA. Evolving epidemiology of HIV-associated malignancies. Curr Opin HIV AIDS. 2017;12(1):6-11.

40. Franzetti M, Ricci E, Bonfanti P. The pattern of non-AIDS-defining cancers in the HIV population: epidemiology, risk factors and prognosis. A review Curr HIV Res. 2019;17:1-12 This study suggests that elevated insomnia and depressive symptoms may partly explain the correlation between HIV serostatus and shorter LTL.

41. Ding Y, Lin H, Zhou S, Wang K, Li L, Zhang Y, et al. Stronger association between insomnia symptoms and shorter telomere length in old HIV-positive patients compared with uninfected individuals. Aging Dis. 2018;9(6):1010-9.

42. Ren J, Zhao M, Liu B, Wu Q, Hao Y, Jiao M, et al. Factors associated with sleep quality in HIV. J Assoc Nurses AIDS Care. 2018;29(6):924-31.

43. Huang X, Li H, Meyers K, Xia W, Meng Z, Li C, et al. Burden of sleep disturbances and associated risk factors: a cross-sectional survey among HIV-infected persons on antiretroviral therapy across China. Sci Rep. 2017;7:3657.

44. Ning C, Lin H, Chen X, Qiao X, Xu X, Xu X, et al. Cross-sectional comparison of various sleep disturbances among sex- and agematched HIV-infected versus HIV-uninfected individuals in China. Sleep Med. 2020;65:18-25.

45. Shan S, Shi Y, Ye R, Yang Y, Yang S, Tang R, et al. A crosssectional survey of food intake frequency and dietary pattern among HIV/AIDS patients receiving antiretroviral therapy in Dehong prefecture, Yunnan province. Chin J AIDS \& STD. 2017;23:116-9.

46. Liu Y, Lv Q, Gao J, Long L, Duan Z, Liang H, et al. Coinfection with HIV-1 alleviates iron accumulation in patients with chronic hepatitis C virus infection. PLoS One. 2014;9:e98039.

47. Ji G, Qi R, Wang H, Feng C, Leng J. A "planting and eating soybean" project for people living with HIV/AIDS in rural Anhui-a pilot study in China. AIDS Care. 2010;22:126-32.

48. Zhou W, Ma R, Sharma M, Zhao Y. Nutritional attitudes of homosexual people living with human immunodeficiency virus (HIV) in Chongqing, Southwest China: a cross-sectional study. Int Q Community Health Educ 2013-2014; 34:87-100.
49. Ipp H, Zemlin A. The paradox of the immune response in HIV infection: when inflammation becomes harmful. Clin Chim Acta. 2013;416:96-9.

50. Nou E, Lo J, Grinspoon SK. Inflammation, immune activation, and cardiovascular disease in HIV. AIDS. 2016;30(10):1495-509.

51. Hileman CO, Funderburg NT. Inflammation, immune activation, and antiretroviral therapy in HIV. Curr HIV/AIDS Rep. 2017;14(3):93-100.

52. Sun J, Su J, Xie Y, Yin M, Huang Y, Xu L, et al. Plasma IL-6/IL-10 ratio and IL-8, LDH, and HBDH level predict the severity and the risk of death in AIDS patients with pneumocystis pneumonia. J Immunol Res. 2016;2016:1583951.

53. Zhao D, Ding Y, Lin H, Chen X, Shen W, Gao M, et al. Mitochondrial haplogroups N9 and G are associated with metabolic syndrome among human immunodeficiency virus-infected patients in China. AIDS Res Hum Retrovir. 2019;35(6):536-43.

54. Simoni JM, Safren SA, Manhart LE, Lyda K, Grossman CI, Rao D, et al. Challenges in addressing depression in HIV research: assessment, cultural context, and methods. AIDS Behav. 2011;15(2):37688.

55. Li L, Ji G, Liang LJ, Lin C, Hsieh J, Lan CW, et al. Efficacy of a multilevel intervention on the mental health of people living with HIV and their family members in rural China. Health Psychol. 2017;36(9):863-71.

56. Diaz RM, Ayala G, Bein E, Henne J, Marin BV. The impact of homophobia, poverty, and racism on the mental health of gay and bisexual Latino men: findings from 3 US cities. Am J Public Health. 2001;91(6):927-32.

57. Brown JR, O'Connor GT. Coronary heart disease and prevention in the United States. N Engl J Med. 2010;362(23):2150-3.

58. Galea S, Ahern J, Nandi A, Tracy M, Beard J, Vlahov D. Urban neighborhood poverty and the incidence of depression in a population-based cohort study. Ann Epidemiol. 2007;17(3):171-9.

59. Singer M. Introduction to syndemics: a critical systems approach to public and community health. John Wiley \& Sons; 2009.

60. Stall R, Mills TC, Williamson J, Hart T, Greenwood G, Paul J, et al. Association of co-occurring psychosocial health problems and increased vulnerability to HIV/AIDS among urban men who have sex with men. Am J Public Health. 2003;93(6):939-42.

Publisher's Note Springer Nature remains neutral with regard to jurisdictional claims in published maps and institutional affiliations. 